\title{
Comparison of the therapeutic effects of the dietary and topical forms of Zizyphus jujuba extract on oral mucositis induced by 5-fluorouracil: A golden hamster model
}

\author{
Omid Koohi-Hosseinabadi ${ }^{1}$, Azadeh Andisheh-Tadbir ${ }^{2}$, Parisa Bahadori ${ }^{3}$, Masood Sepehrimanesh ${ }^{4}$, Maryam \\ Mardani ${ }^{5}$, Nader Tanideh ${ }^{6}$ \\ ${ }^{1}$ Laboratory Animals Center, Shiraz University of Medical Sciences, Shiraz, Iran \\ ${ }^{2}$ Department of Oral and Maxillofacial Pathology, School of Dentistry, Shiraz University of Medical Sciences, Shiraz, Iran \\ ${ }^{3}$ Students' Research Committee, School of Dentistry, International Branch, Shiraz University of Medical Sciences, Shiraz, Iran \\ ${ }^{4}$ Gastroenterohepatology Research Center, Shiraz University of Medical Sciences, Shiraz, Iran \\ ${ }^{5}$ Department of Oral Medicine, School of Dentistry, Shiraz University of Medical Sciences, Shiraz, Iran \\ ${ }^{6}$ Stem Cell and Transgenic Technology Research Center and Department of Pharmacology, School of Medicine, Shiraz University \\ of Medical Sciences, Shiraz, Iran
}

Correspondence:

Department of Pharmacology

School of Medicine

Shiraz University of Medical Sciences

PO. Box: 7134845794, Shiraz, Iran

nadertanideh@yahoo.com

Koohi-Hosseinabadi O, Andisheh-Tadbir A, Bahadori P, Sepehrimanesh M, Mardani M, Tanideh N. Comparison of the therapeutic effects of the dietary and topical forms of Zizyphus jujuba extract on oral mucositis induced by 5-fluorouracil: A golden hamster model. J Clin Exp Dent. 2015;7(2):e304-9.

Received: $17 / 11 / 2014$

Accepted: 28/12/2014

http://www.medicinaoral.com/odo/volumenes/v7i2/jcedv7i2p304.pdf

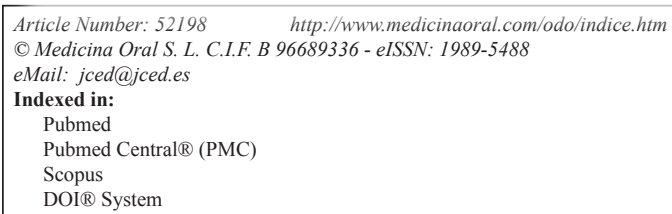

\begin{abstract}
Background: Oral mucositis $(\mathrm{OM})$ is a common inflammatory complication among cancerous patients as an adverse effect of chemotherapy and radiotherapy. The aim of this study was to evaluate the effects and identify the appropriate route of administration of extract of Zizyphus jujuba in 5-flurouracile induced OM induction in golden hamster.

Materials and Methods: In a prospective randomized double blind animal study, OM was induced in 56 male golden hamsters by 5 -FU $(60 \mathrm{mg} / \mathrm{kg})$ on days 0,5 , and 10 of the study. The cheek pouch was scratched with a sterile needle on once daily on days 3 and 4 . On days $14-17,300 \mathrm{mg} / \mathrm{kg}$ dietary and $20 \%$ Z jujuba gel and gel base groups were treated and then compared with a control group. Microscopic scores and pouch content of malondialdehyde (MDA), plus activities of superoxide dismutase and myeloperoxidase in pouch tissue were evaluated.

Results: Histopathology scores of mucositis were lower in the systemic and $20 \%$. jujuba gel groups than the gel base and control groups $(P<0.05)$. Also, lower MDA level and higher activities of MPO and SOD were detected in the systemic and $20 \% Z$. jujuba gel groups in comparison to the gel base and control groups $(P<0.001)$.

Conclusions: The use of $Z$. jujuba hydroalcoholic extract in systemic and topical forms may be associated with reduced intensity of OM, diminished concentration of MDA, and increased activity of MPO and SOD on induced OM in golden hamster undergoing 5-FU consumption.
\end{abstract}

Key words: Oral mucositis, 5-flurouracil, Zizyphus jujube, oxidative stress, histopathology score. 


\section{Introduction}

Inflammation is a physiological response of body especially vascular tissues to harmful stimuli, including infections and tissue injury. As the primary interface between the body and the external environment, the skin provides the first line of defense against traumatic injury and invasion by microbial pathogens (1). On the other hand, for orally digested chemical agents and microbiota, oral mucosa is the first line of defense and approximately in all cases, it suffer histopathological and inflammatory injuries. Oral mucositis (OM) was first described in 1980s and is the painful inflammation and ulceration of the mucous membranes lining the oral cavity, usually as an adverse effect of treatment for cancer (2). Both chemotherapy and radiation therapy can interfere with maturity, cellular growth, and renewal rate of basal epithelial cells, causing changes to normal turnover and cell death (3). Indeed, OM is the result of a series of inflammatory changes in the epithelial and subepithelial cells of the oral mucosa and is a debilitating toxicity of chemoradiotherapy for head and neck cancer (HNC) (4, 5). $\mathrm{HNC}$, principally squamous cell carcinoma, is one of the main oncological problems owing to its high mortality rate and the side-effects of the treatment (5). One of the chemotherapeutic drugs that can be induced OM is fluorouracil (5-FU). 5-FU, which trademarked as Adru$\operatorname{cil}{ }^{\circledR}, \operatorname{Carac} \AA$ and Efudex $\AA$, is a pyrimidine analog drug which is used in the treatment of cancer (6). 5-FU has been administered systemically for anal, breast, colorectal, esophageal, stomach, pancreatic and skin cancers (especially HNC) and topically for actinic keratoses and Bowen's disease (3).

Management of OM via topical approaches should address efficacy, patient acceptance, and appropriate dosing. Although, we conducted some reports about using dexamethasone as chemical drugs for prevention of $\mathrm{OM}$ $(7,8)$, but medicinal plants have attracted more attention. A large number of plant species contain various bioactive compounds exhibiting health beneficial properties, anti-oxidative, anti-inflammatory and mainly antimicrobial effects, and their preventive and therapeutic use increases (1). In our point of view, it has been reported that Aloe vera mouthwash may reduce radiation-induced $\mathrm{OM}$ in HNC patients (9). Also, our previous studies demonstrated that some medicinal plants such as bilberry (10), St. John's wort (2) and Marigold (3) could effectively intervene against development of OM.

Zizyphus jujuba is a thorny rhamnaceous plant that is widely distributed in Europe and Southeastern Asia (11). Pharmacologically active peptides including cyclopeptide alkaloids, flavonoids, sterols, jujuboside A, jujuboside $\mathrm{B}$, lauric acid, and triterpenoid saponins have been isolated and chemically identified from different species of $Z$. jujuba (12). Fruits of this plant are edible and different parts of $Z$. jujuba possess multiple medicinal pro- perties such as antifertility, analgesic, and antidiabetes (11). It also has been used as tranquilizer, analgesic, and anticonvulsant in folk medicine in India (13). However, there are no scientific studies about the effect of $Z$. juju$b a$ fruit in prevention and treating of OM. Considering the debilitating conditions that OM induced by chemoradiotherapy can cause in cancer patients and the lack of a definite treatment strategy, and based on the data implicating the anti-inflammatory, analgesic and wound healing activities of $Z$. jujube, we decided to evaluate and compare the effects of hydroalcoholic extract of the fruit of Z. jujuba in two forms, topical and dietary, on 5 -FU induced $\mathrm{OM}$ in golden hamster.

\section{Material and Methods}

\section{-Animals and housing}

This study was approved by the Research and Ethics Committee of Shiraz University of Medical Sciences, and all relevant considerations about animal rights were taken into this study. This randomized animal trial was performed in the Laboratory Animal Center of Shiraz University of Medical Sciences. Fifty six male golden hamsters (100 to $120 \mathrm{~g}, 6-8$ weeks old) were taken from Laboratory Animal Center of Shiraz University of Medical Sciences, Shiraz, Iran and were housed in standard cages under a 12-h light cycle (lights on at 7:00 pm) with an ambient temperature of $22 \pm 2{ }^{\circ} \mathrm{C}$, and $55 \%$ relative humidity. Hamsters were given a 3-day acclimation period with an access to normal chow and water ad libitum before the experiment. During their stay in the respective housing conditions, they were removed from their cages every 3 days for cleaning the cages and renewing their food and water supply. During the experiment, access to standard laboratory diet and water was allowed ad libitum.

-Preparation of hydroalcoholic extract

The fruit of Z. jujube was collected from Shiraz in the Fars province of Iran. The fruits were dried at room temperature and powdered in a grinder. Aqueous ethanol (75\%) was added to the powdered materials (500 g), and stirred for one hour. The mixture was kept at room temperature for 48 hours. Following filtration, ethanol was evaporated under reduced pressure at $40^{\circ} \mathrm{C}$. The remained water extract was dried at oven temperature of $50^{\circ} \mathrm{C}$. Finally, $20 \%$ Z. jujube hydroalcoholic extract was prepared in pharmacy laboratory.

-Chemotherapy induced OM

Fifty six adult male golden hamsters were randomly allocated into four groups (14 hamsters in each group). All animals received three intraperitoneal injections of 5-FU on days 0,5 , and 10 at a dose of $60 \mathrm{mg} / \mathrm{kg}$ with insulin needles following the protocol proposed by Sonis et al. (14). To mimic the clinical effect of chronic irritation, the cheek pouch mucosa was scratched superficially with the tip of an 18-gauge sterile needle after anestheti- 
zing the area. Two horizontal linear scratches were made across the everted cheek pouch once daily on days 3 and 4. The treatment was started on day 12 and 1 st group of hamsters served as controls and didn't receive any treatment; group 2 received gel base used in preparation of the topical gel; group 3 were treated with topical $Z$. jujuba extract gel $20 \%$ on the cheek pouch mucosa of both sides once a day (morning) and the affected area was completely covered. Group 4 were fed Z. jujuba hydroalcoholic extract with dose $300 \mathrm{mg} / \mathrm{kg}$ once a day (morning) in dietary root during study. To make sure that it was not swallowed, animals weren't allowed to eat or drink for 30 minutes after the drug applications. Animals were weighed daily. Seven hamsters from each group were randomly selected for killing on days 14, and 17 . At the time of sacrifice, they were anesthetized with 100 $\mathrm{mg} / \mathrm{kg}$ ketamine 10\% (Alfasan, Netherlands) and $10 \mathrm{mg} /$ $\mathrm{kg}$ xylasine $2 \%$ (Alfasan, Netherlands).

-Histopathological study

The both side cheek pouch tissue of hamsters in all groups were harvested on day 14 and 17. Tissue obtained from the right side pouch was proceed and stained with hematoxylin-eosin according to previous reported procedure (15-17). The specimens were examined histopathologically and received scores of 0 to 3 , as follows (18):

- Score 0, normal epithelium and connective tissue without vasodilatation; absent or mild inflammatory infiltrate; absence of bleeding, ulcers and abscesses

- Score 1, mild vascular hyperemia; areas of reepithelialization; mild inflammatory infiltrate with a prevalence of mononuclear cells; no hemorrhagic areas, ulcerations or abscesses

- Score 2, moderate vascular redness; areas of epithelial degeneration; inflammatory infiltration with prevalence of neutrophils; hemorrhagic areas, edema and occasional ulcerations; absence of abscesses

- Score 3, severe hyperemia and vascular vasodilatation; inflammatory infiltration

-Measurement of malondialdehyde (MDA)

Tissues obtained from the left side pouch were stored under liquid nitrogen until measurment. Tissue malondialdehyde (MDA) assessment was performed via the measurement of thiobarbituric acid reactive substances (TBARS) in PBS tissue homogenate (19), because MDA is one of the end-products of lipid peroxidation (LPO), and the extent of LPO is most frequently measured by estimating MDA levels (20).

-Superoxide dismutase (SOD) assay

Total SOD activity was evaluated with SOD detection kit (BiorexFars, Iran) according to the manufacturer's instructions. The role of SOD is to accelerate the dismutation of the toxic superoxide $\left(\mathrm{O}_{2}^{-}\right)$produced during oxidative energy processes to hydrogen peroxide and molecular oxygen. This method employs xanthine and xanthine oxidase (XOD) to generate superoxide radicals which react with 2-(4-iodophenyl)-3-(4-nitrophenol)-5phenyltetrazolium chloride (INT) to form a red formazan dye. The SOD activity is then measured by degree of inhibition of this reaction. One unit of SOD is that which causes $50 \%$ inhibition of the rate of reduction of INT under the conditions of the assay. SOD levels were recorded at $505 \mathrm{~nm}$ and through a standard curve and expressed as $\mathrm{U} / \mathrm{g}$ wet tissue.

-Myeloperoxidase (MPO) assay

MPO activity can be measured in tissues by assays using hydrogen peroxide and o-dianisidine dihydrochloride as substrates (21). Briefly, cheek pouch tissues which collected on days 14 and 17, were weighed and homogenized in a solution containing $0.5 \%(\mathrm{w} / \mathrm{v})$ hexadecyltrimethylammonium bromide dissolved in $10 \mathrm{mmol} / \mathrm{L}$ potassium phosphate buffer $(\mathrm{pH} 7.4)$ and centrifuged for 30 minutes at $20,000 \mathrm{~g}$ at $4^{\circ} \mathrm{C}$. An aliquot of supernatant was then removed and added to a reaction mixture containing $1.6 \mathrm{mmol} / \mathrm{L}$ tetramethylbenzidine and 0.1 $\mathrm{mmol} / \mathrm{L}$ hydrogen peroxide $\left(\mathrm{H}_{2} \mathrm{O}_{2}\right)$. The rate of change in absorbance was measured spectrophotometrically at $650 \mathrm{~nm}$. MPO activity was defined as the quantity of enzyme required to degrade $1 \mathrm{mmol}^{\circ} \mathrm{H}_{2} \mathrm{O}_{2}$ at $37^{\circ} \mathrm{C}$ and was expressed in $\mathrm{U} / \mathrm{g}$ wet tissue.

-Statistical analysis

All statistical analyses were carried out using SPSS ${ }^{\circledR}$ statistical software, version 20.0 (SPSS Inc., Chicago, IL, USA) for Windows ${ }^{\circledR}$. Data are expressed as the mean \pm standard deviation (SD) from all independent experiments. One-Sample-Kolmogorov-Smirnov test was used to test for normality of distribution of the collected data. A two tailed paired sample $\mathrm{T}$ tests were used to compare MDA level, SOD and MPO activities and pathology score between days 14 and 17 in control group and treatment groups. Differences were considered significant when $P<0.05$.

\section{Results}

-Histopathology evaluation

The maximum severity of OM was seen on day 12 (base on clinical examination) and the treatment thus was started on this day. There is no histopathologic score (HPS) difference between the gel base and control groups and between the topical gel and systemic treatment groups on both days. Also, only significant decreases are seen after 5 days treatment in comparison to 2 days treatment in the HPS in response to topical gel and systemic treatment groups $(P<0.05)$. These declines indicating less severe inflammation in the topical gel and systemic treatment groups (Fig. 1). Epithelialization and wound healing were detected in the topical gel and systemic treatment groups, whereas extensive infiltration of inflammatory cells, hemorrhage and ulcer were observed in the control and gel base groups. On days 12 and 17, HPS for the topical gel and systemic treatment groups were less than 

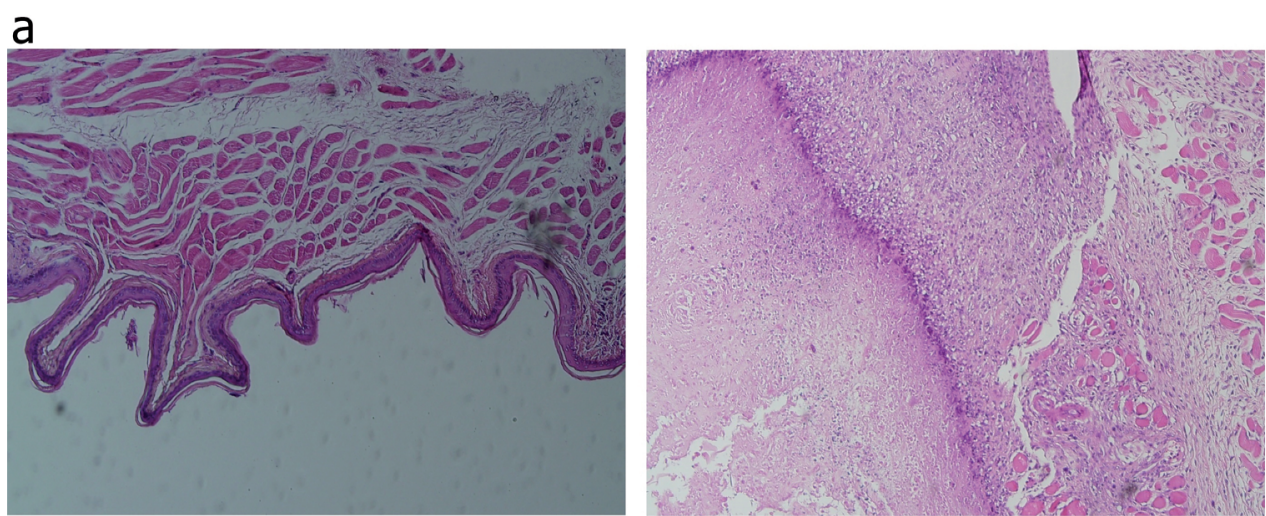

b

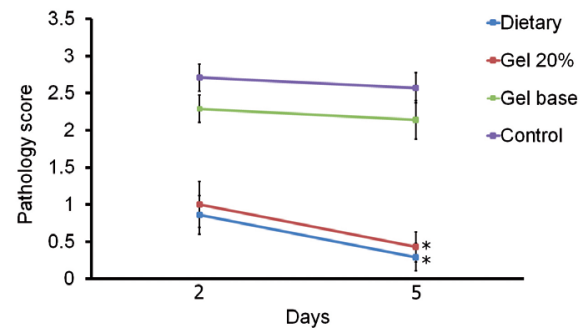

Fig. 1. The histopathology lesions a) and score b) comparison between four different groups in two days of sampling. In histopathological slides, left is score 0 and right is score 3. Significant differences $(P<0.05)$ between two days were indicated by asterisk.

gel base group $(P<0.001)$. Also on these two days, HPS for the topical gel and systemic treatment groups were less than that for the control group $(P<0.001)$.

-Tissue MDA level

Tissue MDA concentration in experimental groups on 2 and 5 days after onset of treatment are shown in figure 2. There are significant differences between MDA concentrations in all four groups between days 2 and 5. Also, MDA concentration is higher in the control and gel base groups than in the topical gel and systemic treatment groups on all days $(P<0.001)$. Although, MDA concentration of the topical gel was higher than the systemic treatment group on day $14(P=0.026)$, but on day 17, MDA level in the topical gel was lower than the systemic treatment group $(P=0.728)$. Totally, both systemic and topical treatment could diminish the MDA level obviously.

-Tissue MPO activity

Tissue MPO activity in experimental groups on 2 and 5 days after onset of treatment are shown in figure 3. There are significant differences between MPO activity in all four groups between days 2 and 5. Also, MPO had more activity in the systemic treatment and the topical gel groups than in the control and gel base groups on all days $(P<0.001)$. Difference between the control and gel base groups and also between the systemic treatment and the topical gel groups on day 14 ( 2 days after treatment) were not significant ( $P=0.987$ and $P=0.180$, respectively). On day 17 ( 5 days after treatment) significant differences were detected between the systemic treatment and the topical

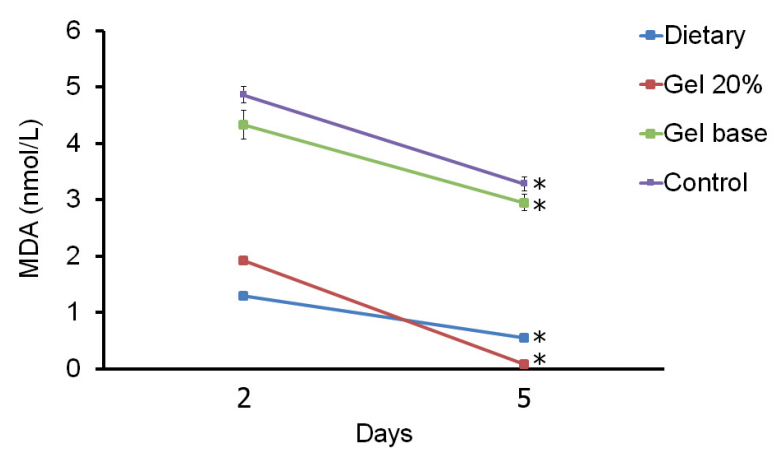

Fig. 2. The mean $\pm \mathrm{SD}$ comparison of malondialdehyde (MDA) concentration between four different groups in two days of sampling. Significant differences $(P<0.05)$ between two days were indicated by asterisk.

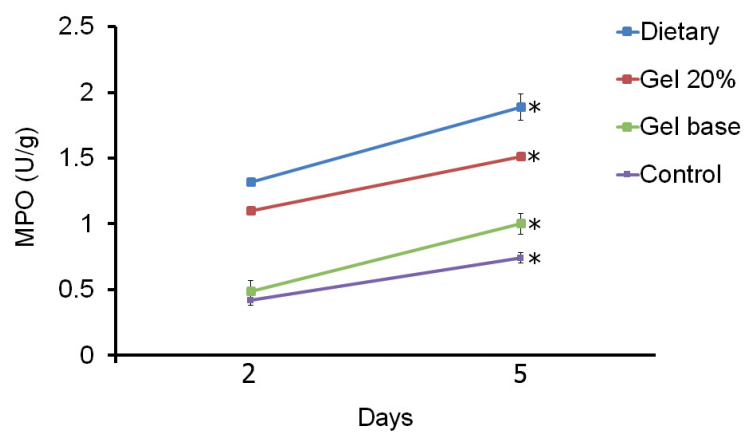

Fig. 3. The mean \pm SD comparison of myeloperoxidase (MPO) activity between four different groups in two days of sampling. Significant differences $(P<0.05)$ between two days were indicated by asterisk. 
gel groups $(P=0.001)$, between the systemic treatment and the control and gel base groups $(P<0.001)$ and between the topical gel and the control and gel base groups $(P<0.001)$. On day 17 , there was no significant difference between the control and gel base groups $(P=0.058)$.

-Tissue SOD activity

Tissue SOD activity in experimental groups on 2 and 5 days after onset of treatment are shown in figure 4 . There are significant differences between SOD activity only in the systemic treatment and the topical gel groups between days 2 and 5. SOD had more activity in the systemic treatment and the topical gel groups than the control and gel base groups on all days $(P<0.001)$. Although, difference between the control and gel base groups on day 14 ( 2 days after treatment) was not significant $(P=0.996)$, but on this day, the systemic treatment and the topical gel groups showed significant difference $(P<0.001)$. On day 17 (5 days after treatment) significant differences were detected between the systemic treatment and the topical gel groups $(P=0.001)$, between the systemic treatment and the control and gel base groups $(P<0.001)$ and between the topical gel and the control and gel base groups $(P<0.001)$. On day 17 , there was no significant difference between the control and gel base groups $(P=0.222)$.

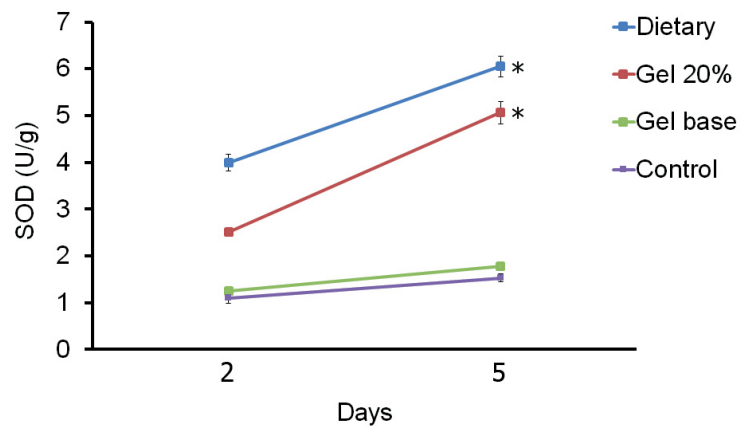

Fig. 4. The mean $\pm \mathrm{SD}$ comparison of superoxide dismutase (SOD) activity between four different groups in two days of sampling. Significant differences $(P<0.05)$ between two days were indicated by asterisk.

\section{Discussion}

This study compared the effects of two different drug forms of $Z$. jujuba extract, topical gel $20 \%$ and $300 \mathrm{mg} /$ $\mathrm{kg}$ dietary forms, on the healing of OM induced by 5-FU in golden hamster. Our results demonstrated that all two drug forms can induce wound healing by decreasing MDA concentration and elevating MPO and SOD activity. Also, the systemic dietary form was approximately more potent than topical gel.

Although, inflammatory diseases are currently treated with steroidal and non-steroidal anti-inflammatory drugs (NSAIDs) (1), but, there are several reports about the healing and anti-inflammatory effects of recombinant proteins and medicinal plants in OM, as an inflamma- tory disease of oral mucosa, in human and animal models $(2-4,10,18,22-25)$. OM is the most prevalent side effect of the anticancer treatment, accounting for more than $90 \%$ of patients receiving radiotherapy to treat HNC (26). In two randomized, placebo-controlled trials conducted in $\mathrm{HNC}$ patients undergoing postoperative chemoradiotherapy and in patients receiving definitive chemoradiotherapy for locally advanced HNC, intravenous palifermin, a truncated human recombinant keratinocyte growth factor (KGF), administered weekly for 8 weeks decreased severe $\mathrm{OM}(4,23)$. On the point of medicinal plant view, the effect of essential oil from seeds of Z. jujube on 12-O-tetradecanoylphorbol-13-acetate (TPA) induced skin inflammation in experimental mice was investigated by Al-Reza and his colleagues. They reported that $Z$. jujuba essential oil inhibited the inflammatory responses of skin inflammation in animal model. Also, they concluyded that the essential oil of $Z$. jujuba seeds might accelerate the development of new drugs for various inflammatory diseases (1). Also, the use of mouthwash containing 1\% Chamomilla recutita extract could be associated with reduced incidence, intensity, and duration of mucositis in adults patients undergoing allogenic hematopoietic stem cell transplantation which suffered from OM (22). In another study, the effectiveness of Matricaria recutita Linn (chamomile) in the prevention and control of radiation-induced OM was evaluated. Their results showed that group with chamomile gel had the faster clinical improvement (24). In all previous reports, plants and their extracts had no prophylactic effect on the onset of $\mathrm{OM}$, but it was proven to be effective in decreasing the severity of this condition. Also, it has been reported that the beneficial effects of these traditional plants may be due to polyphenols content which reviewed by Varoni et al. (25).

On the other hand, oxidative injury from free radical generation is involved in the pathophysiology of many human diseases (13). Several investigations have been performed to elucidate the exact role of highly-specific molecules or mechanisms underlying the OM development $(7,18,27)$. It has been demonstrated that hydroalcoholic extract of $Z$. jujube can reverse oxidative stress induced by pentylenetetrazole (PTZ) and electroshock (13). Our results rendered more evidence to the critical role of free radicals and protective roles of antioxidative enzymes included SOD and MPO in pathogenesis of OM. These protective effects are useful seen in the groups receiving systemic and topical forms of Z. jujube.

\section{Conclusions}

According to the findings of present study, Z. jujube might be effective agent in treatment of OM. However, further studies must be performed on the finding of its active anti-inflammatory and antioxidative components in different diseases and clinical trials. 


\section{References}

1. Al-Reza SM, Yoon JI, Kim HJ, Kim JS, Kang SC. Anti-inflammatory activity of seed essential oil from Zizyphus jujuba. Food Chem Toxicol. 2010;48:639-43.

2. Tanideh N, Namazi F, Andisheh Tadbir A, Ebrahimi H, Koohi-Hosseinabadi O. Comparative assessment of the therapeutic effects of the topical and systemic forms of Hypericum perforatum extract on induced oral mucositis in golden hamsters. Int J Oral Maxillofacial Surg. 2014;43:1286-92.

3. Tanideh N, Tavakoli P, Saghiri MA, Garcia-Godoy F, Amanat D, Andisheh Tadbir A, et al. Healing acceleration in hamsters of oral mucositis induced by 5 -fluorouracil with topical Calendula officinalis. Oral Surg Oral Med Oral Pathol Oral Radiol. 2013;115:332-8.

4. Le QT, Kim HE, Schneider CJ, Murakozy G, Skladowski K, Reinisch S, et al. Palifermin reduces severe mucositis in definitive chemoradiotherapy of locally advanced head and neck cancer: a randomized, placebo-controlled study. J Clin Oncol. 2011;29:2808-14.

5. Rodríguez-Caballero A, Torres-Lagares D, Robles-Garcia M, Pachon-Ibanez J, Gonzalez-Padilla D, Gutierrez-Perez JL. Cancer treatment-induced oral mucositis: a critical review. Int J Oral Maxillofacial Surg. 2012;41:225-38.

6. Gamelin E, Boisdron-Celle M. Dose monitoring of 5-fluorouracil in patients with colorectal or head and neck cancer-status of the art. Crit Rev Oncol Hematol. 1999;30:71-9.

7. Davarmanesh M, Mardani M, Mirkhani H, Monabbati A, Tanideh N, Mehrabani D. Effect of dexamethasone premedication on oral mucositis in hamster. J Appl Anim Res. 2009;36:267-70.

8. Davarmanesh M, Mardani M, Mirkhani H, Monabati A, Tanideh N, Khalgholah M. Effect of systemic dexamethasone premedication on short-term experimentally Induced oral mucositis in hamsters. J Dentist Shiraz Uni Med Sci. 2007:7:83-95.

9. Ahmadi A. Potential prevention: Aloe vera mouthwash may reduce radiation-induced oral mucositis in head and neck cancer patients. Chin J Integrat Med. 2012;18:635-40.

10. Davarmanesh M, Miri R, Haghnegahdar S, Andishe Tadbir A, Tanideh N, Saghiri MA, et al. Protective effect of bilberry extract as a pretreatment on induced oral mucositis in hamsters. Oral Surg Oral Med Oral Pathol Oral Radiol. 2013;116:702-8.

11. Yoon J, Al-Reza SM, Kang SC. Hair growth promoting effect of Zizyphus jujuba essential oil. Food Chem Toxicol. 2010;48:1350-4.

12. Liu J, Chen B, Yao S. Simultaneous analysis and identification of main bioactive constituents in extract of Zizyphus jujuba var. sapinosa (Zizyphi spinosi semen) by high-performance liquid chromatography-photodiode array detection-electrospray mass spectrometry. Talanta. 2007;71:668-75. 13. Pahuja M, Mehla J, Reeta KH, Joshi S, Gupta YK. Hydroalcoholic extract of Zizyphus jujuba ameliorates seizures, oxidative stress, and cognitive impairment in experimental models of epilepsy in rats. Epilepsy Behavior. 2011;21:356-63.

14. Sonis ST, Tracey C, Shklar G, Jenson J, Florine D. An animal model for mucositis induced by cancer chemotherapy. Oral Surg Oral Med Oral Pathol. 1990;69:437-43.

15. Khodakaram Tafti A, Nazifi S, Rajaian H, Sepehrimanesh M, Poorbaghi SL, Mohtarami S. Pathological changes associated with experimental salinomycin toxicosis in sheep. Comp Clin Pathol. 2008; 17:255-8.

16. Sepehrimanesh M, Azarpira N, Saeb M, Nazifi S, Kazemipour $\mathrm{N}$, Koohi O. Pathological changes associated with experimental 900-MHz electromagnetic wave exposure in rats. Comp Clin Pathol. 2014:23:1629-31.

17. Tamadon A, Nikahval B, Sepehrimanesh M, Mansourian M, Tabatabaei Naeini A, Nazifi S. Epididymis ligation: a minimally invasive technique for preparation of teaser rams. Vet Surg. 2010;39:121-7.

18. Lima V, Brito GAC, Cunha FQ, Reboucas CG, Falcao BAA, Augusto RF, et al. Effects of the tumour necrosis factor- $\alpha$ inhibitors pentoxifylline and thalidomide in short-term experimental oral mucositis in hamsters. Eur J Oral Sci. 2005;113:210-7.

19. Subbarao KV, Richardson JS, Ang LC. Autopsy samples of Alzheimer's cortex show increased peroxidation in vitro. J Neurochem. 1990;55:342-5.
20. Lata H, Ahuja GK, Narang APS, Walia L. Effect of immobilisation stress on lipid peroxidation and lipid profile in rabbits. Indian J Clin Biochem. 2004;19:1-4.

21. Mullane KM, Kraemer R, Smith B. Myeloperoxidase activity as a quantitative assessment of neutrophil infiltration into ischemie myocardium. J Pharmacol Meth. 1985;14:157-67.

22. Braga FT, Santos AC, Bueno PC, Silveira RC, Santos CB, Bastos $\mathrm{JK}$, et al. Use of Chamomilla recutita in the prevention and treatment of oral mucositis in patients undergoing hematopoietic stem cell transplantation: A randomized, controlled, phase II clinical trial. Cancer Nursing. 2014 Sep 17. [Epub ahead of print]

23. Henke M, Alfonsi M, Foa P, Giralt J, Bardet E, Cerezo L, et al. Palifermin decreases severe oral mucositis of patients undergoing postoperative radiochemotherapy for head and neck cancer: a randomized, placebo-controlled trial. J Clin Oncol. 2011;29:2815-20.

24. Holmes TSV, Pereira JV, de Castro Gomes DQ, Pereira MdSV, de Medeiros Nóbrega DR, dos Santos MGC. Evaluation of the effectiveness of Matricaria recutita Linn. in the prevention and control of radiation-induced oral mucositis. J Dent Sci. 2014;28:89-93.

25. Varoni EM, Lodi G, Sardella A, Carrassi A, Iriti M. Plant polyphenols and oral health: old phytochemicals for new fields. Curr Med Chem. 2012;19:1706-20.

26. Epstein JB, Klasser GD. Emerging approaches for prophylaxis and management of oropharyngeal mucositis in cancer therapy. Expert Opin Emerg Drugs. 2006;11:353-73.

27. Sonis ST. The biologic role for Nuclear Factor-KappaB in disease and its potential involvement in mucosa injury associated with antineoplastic therapy. Crit Rev Oral Biol Med. 2002;13:380-9.

\section{Acknowledgements}

This paper has been extracted from Ms Parisa Bahadori's DDS thesis which was conducted under supervision of Dr. Azadeh Andisheh Tadbir and Dr. Nader Tanideh and advisory of Dr. Maryam Mardani. The study was approved, registered with ID 8793068 , and supported by the International Branch of Shiraz University of Medical Sciences. 